\title{
MODELING AND SIMULATION OF HIGH RESOLUTION OPTICAL REMOTE SENSING SATELLITE GEOMETRIC CHAIN
}

\author{
Zhongqiu Xia ${ }^{1, *}$, Shaoyuan Cheng ${ }^{1}$, Qiaolin Huang ${ }^{1}$, Guoliang Tian ${ }^{1}$ \\ ${ }^{1}$ Beijing Institute of Space Mechanics \& Electricity, Beijing, China - xiazhongqiu @ hotmail.com
}

Commission III, WG III/1

KEY WORDS: High Resolution, Geometric Chain, Modeling, Simulation, Geolocation Accuracy

\begin{abstract}
:
The high resolution satellite with the longer focal length and the larger aperture has been widely used in georeferencing of the observed scene in recent years. The consistent end to end model of high resolution remote sensing satellite geometric chain is presented, which consists of the scene, the three line array camera, the platform including attitude and position information, the time system and the processing algorithm. The integrated design of the camera and the star tracker is considered and the simulation method of the geolocation accuracy is put forward by introduce the new index of the angle between the camera and the star tracker. The model is validated by the geolocation accuracy simulation according to the test method of the ZY-3 satellite imagery rigorously. The simulation results show that the geolocation accuracy is within $25 \mathrm{~m}$, which is highly consistent with the test results. The geolocation accuracy can be improved about $7 \mathrm{~m}$ by the integrated design. The model combined with the simulation method is applicable to the geolocation accuracy estimate before the satellite launching.
\end{abstract}

\section{INTRODUCTION}

In recent years, the high resolution optical remote sensing satellite has achieved a great progress. The level of the design, the manufacture and the application of the satellite has been improved continuously. The simulation of these processes is becoming more and more important (Poli et al., 2015). In this area, the imaging chain of the satellite system is now in common use (Fiete et al., 1999), which contains two aspects of the radiation and the geometry generally. According to the concept of the radiation chain well known before (Börner et al., 2001), the concept of the geometric chain should be put forward to simulate the geometric properties of the satellite system.

The high resolution satellite with the camera of the longer focal length and the larger aperture has been widely used in obtaining the three dimensional coordinate information of the observed scene recently (Huang et al., 2007), which means the higher requirements of the on-orbit geometric stability of the whole imaging system. The index of geolocation accuracy in plane and high without the ground control points can reflect the whole system geometric performance, which has a significant importance for the satellite performance assessment. To improve the geolocation accuracy, the on-orbit stability index assignment must be reasonable, which can be decided in the simulation based on the geometric chain.

\section{MODELING AND SIMULATION}

\subsection{Modeling of Geometric Chain}

The consistent end to end model of high resolution remote sensing satellite geometric chain is presented, which consists of the observed scene, the three line array camera, the platform including attitude and position information, the time system and the processing algorithm. Besides the parameters including the principal point, the focal length, the distortion of the camera, the attitude and the projective center position of the platform, some new indexes are introduced including the angles among the camera, the satellite and the star tracker. In addition, the integrated design of the camera and the star tracker is considered to validate whether the integrated design can improve the geolocatin accuracy or not.

Considering the distortion, the traditional rigorous model (Poli et al., 2007) can be expressed as

$$
\begin{aligned}
& {\left[\begin{array}{c}
X \\
Y \\
Z
\end{array}\right]_{W G S 84}=\left[\begin{array}{c}
X_{G P S} \\
Y_{G P S} \\
Z_{G P S}
\end{array}\right]_{W G S 84}+R_{J 2000}^{W G S 84} R_{\text {Orbit }}^{J 2000} R_{\text {Satellite }}^{\text {Orbit }}} \\
& \left(\left[\begin{array}{c}
E_{X} \\
E_{y} \\
E_{z}
\end{array}\right]+\left[\begin{array}{c}
e_{X} \\
e_{y} \\
e_{z}
\end{array}\right]+m R_{\text {Camera }}^{\text {Satellite }}\left[\begin{array}{c}
0 \\
y-y_{0}-D \\
-f
\end{array}\right]\right)
\end{aligned}
$$

where $X, Y, Z$ are the coordinates of the object point in the WGS-84 coordinate system; $X_{G P S}, Y_{G P S}, Z_{G P S}$ are the coordinates of the perspective center in the WGS- 84 coordinate system; $y$ is the coordinate of the corresponding image point; $y_{0}$ is the principal point; $f$ is the focal length; $D$ is the distortion; $m$ is the scaling coefficient; $R_{\text {Camera }}^{\text {Satellite }}$ is the rotation matrix from the camera coordinate system to the satellite coordinate system; $R_{\text {Satellite }}^{\text {Orbit }}$ is the rotation matrix from the satellite coordinate system to the orbit coordinate system; $R_{\text {Orbit }}^{J 2000}$ is

\footnotetext{
* Corresponding author
} 
the rotation matrix from the orbit coordinate system to the J2000 coordinate system; $R_{J 2000}^{W G S 84}$ is the rotation matrix from the $\mathbf{J} 2000$ coordinate system to the WGS-84 coordinate system; $E_{x}, E_{y}, E_{z}$ are three offsets of the GPS antenna phase center in the satellite coordinate system; $e_{x}, e_{y}, e_{z}$ are three offsets of the origin of the camera coordinate system to the origin of the satellite coordinate system.

If the attitude of the satellite in the orbit coordinate system is replaced by the attitude of the star tracker in the inertial coordinate system, the Equation 1 will be

$$
\begin{aligned}
& {\left[\begin{array}{c}
X \\
Y \\
Z
\end{array}\right]_{W G S 84}=\left[\begin{array}{c}
X_{G P S} \\
Y_{G P S} \\
Z_{G P S}
\end{array}\right]_{W G S 84}+R_{J 2000}^{W G S 84} R_{\text {Star }}^{J 2000} R_{\text {Satellite }}^{\text {Star }}} \\
& \left(\left[\begin{array}{c}
E_{X} \\
E_{y} \\
E_{z}
\end{array}\right]+\left[\begin{array}{c}
e_{X} \\
e_{y} \\
e_{z}
\end{array}\right]+m R_{\text {Camera }}^{\text {Satellite }}\left[\begin{array}{c}
0 \\
y-y_{0}-D \\
-f
\end{array}\right]\right)
\end{aligned}
$$

Then when $E_{x}=E_{y}=E_{z}=0$ and $e_{x}=e_{y}=e_{z}=0$, the Equation 2 will be

$$
\left.\begin{array}{l}
{\left[\begin{array}{c}
X \\
Y \\
Z
\end{array}\right]_{W G S 84}=\left[\begin{array}{c}
X_{G P S} \\
Y_{G P S} \\
Z_{G P S}
\end{array}\right]_{W G S 84}+m R_{J 2000}^{W G S 84} R_{\text {Star }}^{J 2000}} \\
R_{\text {Satellite }}^{\text {Star }} R_{\text {Camera }}^{\text {Satellite }} \\
y-y_{0}-D \\
-f
\end{array}\right]
$$

Considering the integrated design of the camera and the star tracker, the Equation 3 can be simplified and the model will be

$$
\left[\begin{array}{l}
X \\
Y \\
Z
\end{array}\right]=\left[\begin{array}{c}
X_{G P S} \\
Y_{G P S} \\
Z_{G P S}
\end{array}\right]+m R_{\text {Star }}^{W G S} R_{\text {Camera }}^{\text {Star }}\left[\begin{array}{c}
0 \\
y-y_{0}-D \\
-f
\end{array}\right]
$$

The influence factors mainly referring to the error of each parameter are summarized in a comprehensive way. The manufacture level is quantified by the systematic error and the degree of system stability is quantified by the stochastic error. The geolocation error can be calculated by the law of error propagation as follow

$$
\left\{\begin{aligned}
\Omega_{X}= & \frac{\partial X}{\partial y_{0}}\left(\varepsilon_{y_{0}}+\Delta_{y_{0}}\right)+\frac{\partial X}{\partial f}\left(\varepsilon_{f}+\Delta_{f}\right)+\frac{\partial X}{\partial D}\left(\varepsilon_{D}+\Delta_{D}\right) \\
& +\frac{\partial X}{\partial X_{S}}\left(\varepsilon_{X S}+\Delta_{X S}\right)+\frac{\partial X}{\partial Y_{S}}\left(\varepsilon_{Y S}+\Delta_{Y S}\right)+\frac{\partial X}{\partial Z_{S}}\left(\varepsilon_{Z S}+\Delta_{Z S}\right) \\
& +\frac{\partial X}{\partial \varphi}\left(\varepsilon_{\varphi}+\Delta_{\varphi}\right)+\frac{\partial X}{\partial \omega}\left(\varepsilon_{\omega}+\Delta_{\omega}\right)+\frac{\partial X}{\partial \kappa}\left(\varepsilon_{\kappa}+\Delta_{\kappa}\right) \\
\Omega_{Y}= & \frac{\partial Y}{\partial y_{0}}\left(\varepsilon_{y_{0}}+\Delta_{y_{0}}\right)+\frac{\partial Y}{\partial f}\left(\varepsilon_{f}+\Delta_{f}\right)+\frac{\partial Y}{\partial D}\left(\varepsilon_{D}+\Delta_{D}\right) \\
& +\frac{\partial Y}{\partial X_{S}}\left(\varepsilon_{X S}+\Delta_{X S}\right)+\frac{\partial Y}{\partial Y_{S}}\left(\varepsilon_{Y S}+\Delta_{Y S}\right)+\frac{\partial Y}{\partial Z_{S}}\left(\varepsilon_{Z S}+\Delta_{Z S}\right) \\
& +\frac{\partial Y}{\partial \varphi}\left(\varepsilon_{\varphi}+\Delta_{\varphi}\right)+\frac{\partial Y}{\partial \omega}\left(\varepsilon_{\omega}+\Delta_{\omega}\right)+\frac{\partial Y}{\partial \kappa}\left(\varepsilon_{\kappa}+\Delta_{\kappa}\right)
\end{aligned}\right.
$$

where $\varepsilon$ expresses the systematic error and the $\Delta$ expresses the random error.

\subsection{Simulation of Geolocation Accuracy}

The ZY-3 satellite three line array camera consists of the forward camera, the nadir forward camera and the backward camera (Tang et al., 2012). The structure scheme is shown in fig.1. Three charge coupled device arrays, each pixel size of which is $7 \mu \mathrm{m}$ are installed on the nadir camera as shown in fig.2. And four charge coupled device arrays, each pixel size of which is $10 \mu \mathrm{m}$ are installed on the forward camera and the backward camera as shown in fig.3.

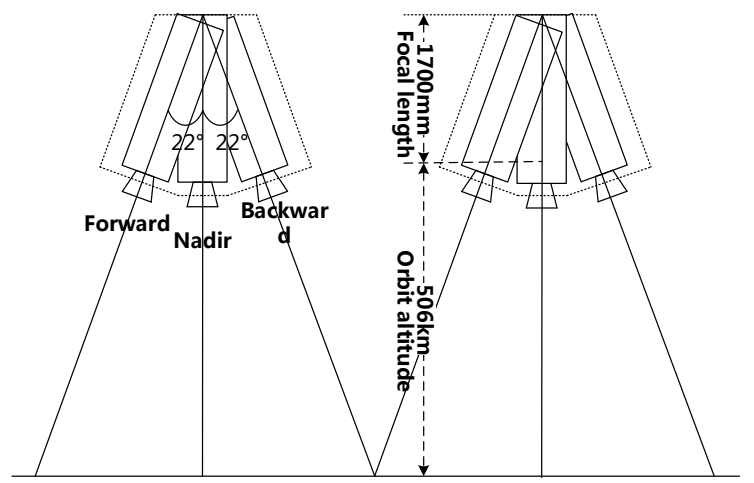

Figure 1. Structure of ZY-3 satellite three line array camera

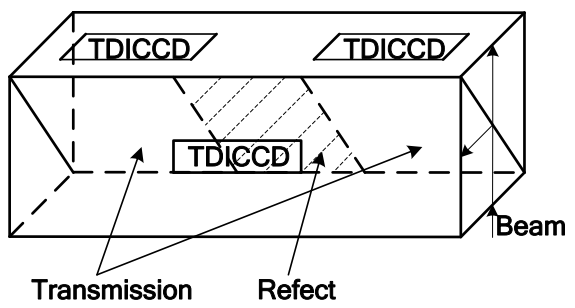

$7 \mu \mathrm{m}$

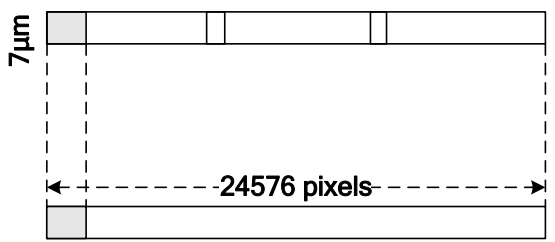

Figure 2. Detectors of nadir camera
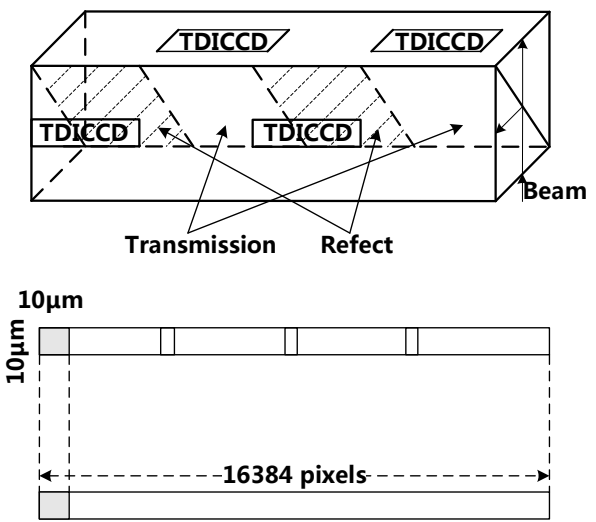

Figure 3. Detectors of forward and backward camera 
Then the simulation method of the geolocation accuracy is proposed referring to the theory of error propagation. The parameters are set as Table.1. To reflect the geometric performance of the whole system, 7 points per line with the same interval are selected to calculate and this operation is repeated 7 times, which is equal to select $7 \times 7$ points of the satellite imagery as the test points.

Table 1. Parameters of simulation

\begin{tabular}{|c|c|c|c|}
\hline \multirow{2}{*}{$\begin{array}{c}\text { Classifi } \\
\text { cation }\end{array}$} & \multirow[b]{2}{*}{ Parameter } & \multicolumn{2}{|c|}{ Design style } \\
\hline & & $\begin{array}{c}\text { Conventional } \\
\text { style }\end{array}$ & $\begin{array}{c}\text { Integrated } \\
\text { style }\end{array}$ \\
\hline \multirow{5}{*}{ Camera } & Width/km & 51.1 & 51.1 \\
\hline & $\begin{array}{l}\text { Principle point } \\
\text { accuracy/ } \mu \mathrm{m}\end{array}$ & 2 & 2 \\
\hline & $\begin{array}{l}\text { Focal length } \\
\text { accuracy } / \mu \mathrm{m}\end{array}$ & 2 & 2 \\
\hline & $\begin{array}{c}\text { Distortion } \\
\text { accuracy } / \mu \mathrm{m}\end{array}$ & 2 & 2 \\
\hline & $\begin{array}{c}\text { Camera install } \\
\text { accuracy/(") }\end{array}$ & 15 & - \\
\hline Orbit & $\begin{array}{c}\text { Orbit determination } \\
\text { accuracy/m }\end{array}$ & 0.05 & 0.05 \\
\hline \multirow{6}{*}{ Attitude } & $\begin{array}{l}\text { Satellite attitude } \\
\text { measurement } \\
\text { accuracy/(") }\end{array}$ & 0.8 & - \\
\hline & $\begin{array}{l}\text { Star track output } \\
\text { frequency/Hz }\end{array}$ & 4 & 4 \\
\hline & $\begin{array}{c}\text { Accuracy of angle } \\
\text { between star track } \\
\text { and camera/(") }\end{array}$ & - & 0.5 \\
\hline & $\begin{array}{l}\text { Satellite attitude } \\
\text { stability } /(\%)\end{array}$ & $5 \times 10^{-4}$ & $5 \times 10^{-4}$ \\
\hline & $\begin{array}{l}\text { Install accuracy of } \\
\text { angle between star } \\
\text { track and camera/(") }\end{array}$ & - & 15 \\
\hline & $\begin{array}{l}\text { Install accuracy of } \\
\text { angle between star } \\
\text { track and satellite/(") }\end{array}$ & 15 & - \\
\hline Time & $\begin{array}{c}\text { Time synchronization } \\
\text { accuracy } / \mu \mathrm{s}\end{array}$ & 20 & 20 \\
\hline Process & $\begin{array}{l}\text { Image point } \\
\text { measurement } \\
\text { accuracy } / \mu \mathrm{m}\end{array}$ & 5 & 5 \\
\hline
\end{tabular}

\section{VALIDATION AND ANALYSIS}

\subsection{Validation Result}

The geometric chain model is validated according to the test method of the ZY-3 satellite imagery rigorously and the simulation of the geolocation accuracy is carried out in four steps. Firstly the error coefficients of each parameter are calculated. Secondly the systematic errors of each parameter are set as 0 considering the systematic errors have been eliminated in the on-orbit calibration of the satellite. Thirdly the residual distribution is analysed to prove the validity of the calibration. At last, the plane geolocation accuracy both in the center and the edge of the field are simulated. As shown in Fig.4, Fig.5 and Fig.6, the systematic errors are eliminated. As shown in Table.2, and the geolocation accuracy is within $25 \mathrm{~m}$, which is highly consistent with the test results (Li et al., 2012).

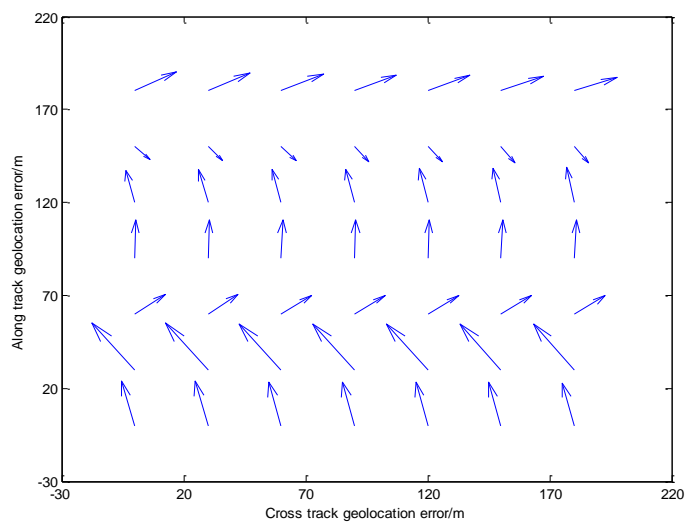

Fig 4 Error distribution of forward camera

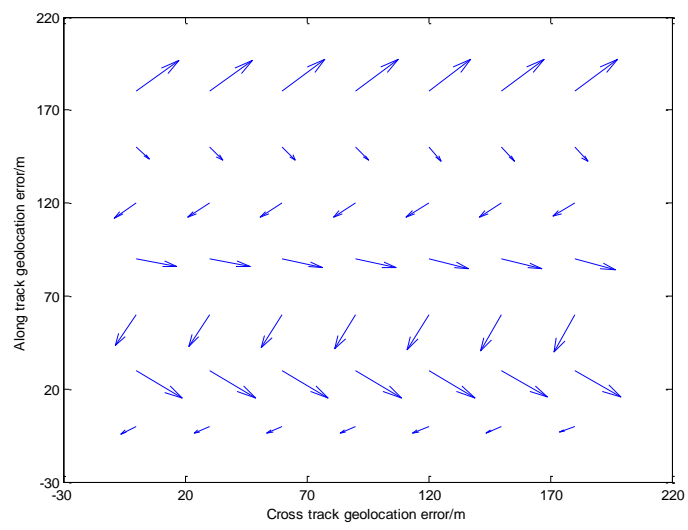

Fig 5 Error distribution of nadir camera

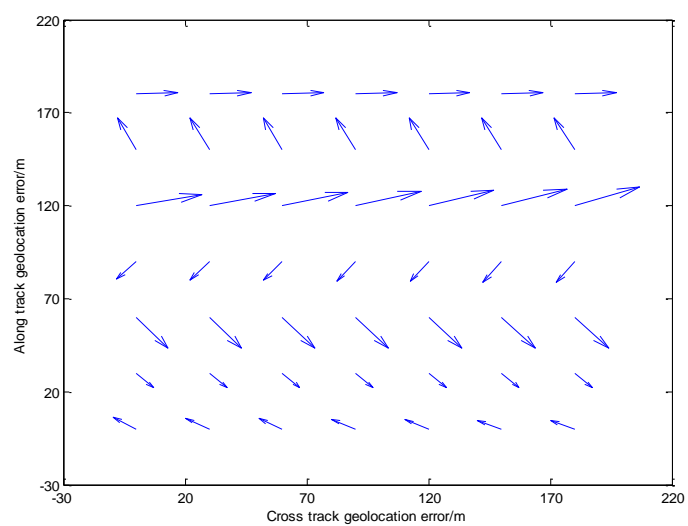

Fig 6 Error distribution of backward camera

Table 2. Geolocation accuracy of conventional model

\begin{tabular}{|c|c|c|c|c|}
\hline \multirow{2}{*}{ Model } & \multirow{2}{*}{$\begin{array}{c}\text { Field } \\
\text { position }\end{array}$} & \multicolumn{3}{|c|}{ Geolocation Accuracy/m } \\
\cline { 3 - 5 } & $\mathrm{X}$ & $\mathrm{Y}$ & Plane \\
\hline $\begin{array}{c}\text { Conventional } \\
\text { model }\end{array}$ & Center & 17.46 & 17.48 & 24.70 \\
\cline { 2 - 5 } & Edge & 17.48 & 17.52 & 24.75 \\
\hline
\end{tabular}

\subsection{Analysis}

Then the geolocation accuracy is also simulated in the condition of the integrated design of the camera and the star tracker. As shown in Table.3, in the center of the field, the geolocation accuracy along the direction of the track can be improved about $5.04 \mathrm{~m}$ from $17.46 \mathrm{~m}$ to $12.42 \mathrm{~m}$ while the 
geolocation accuracy cross the direction of the track can be improved about $5.03 \mathrm{~m}$ from $17.48 \mathrm{~m}$ to $12.45 \mathrm{~m}$, and the plane geoloction accuracy can be improved about $7.11 \mathrm{~m}$ from $24.7 \mathrm{~m}$ to $17.59 \mathrm{~m}$. In the edge of the field, the geolocation accuracy along the direction of the track can be improved about $5.04 \mathrm{~m}$ from $17.48 \mathrm{~m}$ to $12.44 \mathrm{~m}$ while the geolocation accuracy cross the direction of the track can be improved about $5.04 \mathrm{~m}$ from $17.52 \mathrm{~m}$ to $12.48 \mathrm{~m}$, and the plane geoloction accuracy can be improved about $7.13 \mathrm{~m}$ from $24.75 \mathrm{~m}$ to $17.62 \mathrm{~m}$.

Table 3. Geolocation accuracy of integrated model

\begin{tabular}{|c|c|c|c|c|}
\hline \multirow{2}{*}{ Model } & Field & \multicolumn{3}{|c|}{ Geolocation Accuracy/m } \\
\cline { 3 - 5 } & position & $\mathrm{X}$ & $\mathrm{Y}$ & Plane \\
\hline Integrated & Center & 12.42 & 12.45 & 17.59 \\
\cline { 2 - 5 } model & Edge & 12.44 & 12.48 & 17.62 \\
\hline
\end{tabular}

\section{CONCLUSION}

Taking what is mentioned above into account, the on-orbit geometric stability caused by the longer focal length and the larger aperture is a key issue to the high resolution satellite, and the geometric chain model can be used in the analysis of the influence of the satellite stability on the geolocation accuracy. The simulation results based on the geometric chain model are in agreement with the experimental results, and the integrated design of the camera and the star tracker can improve the geolocation accuracy effectively. The geometric chain model combined with the geolocation accuracy simulation method is applicable to the geolocation accuracy estimate before the satellite launching, which can provide an effective way to improve the geolocation accuracy of the high resolution satellite. This is especially benefit for the short cycle and low cost of the design and manufacture of the high resolution satellite.

\section{ACKNOWLEDGEMENTS}

The work was supported by the National Key Research and Development Program of China (No.2016YFB0500802).

\section{REFERENCES}

D. Poli, F. Remondino, E. Angiuli, and G. Agugiaro, 2015. Radiometric and geometric evaluation of GeoEye-1, WorldView-2 and pleiades-1A stereo images for 3D information extraction. ISPRS J. Photogramm. Remote Sens. 100, pp. 35-47.

Börner, A., Wiest, L., Keller, P., Reulke, R., Richter, R., Schaepman, M. and Schläpfer, D., 2001. SENSOR: a tool for the simulation of hyperspectral remote sensing systems, ISPRS Journal of Photogrammetry \& Remote Sensing, 55(1), pp. 299312 .

Fiete, R. D. and Tantalo, A. T., 1999. Image quality of increased along-scan sampling for remote sensing systems, Opt. Eng., 38(5), pp. 815-820.

Huang, Q. L. and Jiang, W., 2007. A Study of the New Direction of Space-borne Hi-resolution Optical Remote Sensor, Spacecraft Recovery \& Remote Sensing, 28(4), pp. 48-50.

T. Jin, Z. Li, T. Li, and D. Yang, 2013. System Design and Analysis for Improving Geometric Accuracy of High-
Resolution Optical Remote Sensing Satellite Image, Journal of Astronautics, 34, pp. 1160-1165.

D. Poli. 2007. A rigorous model for spaceborne linear array sensors. Photogrammetric Engineering \& Remote Sensing, 73(2), pp. 187-196.

Tang, X. M., Zhang, G., Zhu, X. Y., 2012. Triple Linear-array imaging geometry model of Ziyuan-3 surveying satellite and its validation. Acta Geodaetica et Cartographica Sinica, 41(2), pp. 191-198.

Li, D. R., Wang, M., 2012. On-orbit geometric calibration and accuracy assessment of ZY-3. Spacecraft Recovery \& Remote Sensing, 33(3), pp. 1-6. 